R. Greenberg

Nagoya Math. J.

Vol. 89 (1983), 77-87

\title{
ON p-ADIC ARTIN L-FUNCTIONS
}

\author{
RALPH GREENBERG*
}

\section{Introduction}

In this paper we will discuss $p$-adic Artin $L$-functions. The existence of these functions is a simple consequence of a theorem of Deligne and Ribet [4]. One can formulate a "p-adic Artin conjecture" for these functions. Our primary purpose here is to relate this conjecture to the "main conjecture" discussed by Coates in [3]. We will describe the precise formulations of these conjectures that we will use later. Our main result will be that in fact the main conjecture implies the $p$-adic Artin conjecture.

Let $k$ be a totally real number field. Let $\psi$ be an Artin character for $k$. We denote by $k_{\psi}$ the Galois extension of $k$ attached to $\psi$. (Thus, $\psi$ is the character of a faithful representation of $\mathrm{Gal}\left(k_{\psi} / k\right)$.) We will assume throughout this paper that $k_{\psi}$ is also totally real. Otherwise the $p$-adic $L$-function for $\psi$ will be identically zero. Let $k_{\infty}$ denote the cyclotomic $Z_{p}$-extension of $k$. We will say that $\psi$ is of type $S$ if $k_{\psi} \cap k_{\infty}=k$. We can associate to such $\psi$ a purely algebraically defined polynomial $h_{\psi}(T)$. This polynomial will be constructed from a natural representation of Gal $\left(k_{\psi} k_{\infty} / k\right)$ occurring in Iwasawa theory. Its formal properties are quite analogous to those of Artin $L$-functions. Assuming the main conjecture, we can relate this polynomial to the $p$-adic Artin $L$-function for $\psi$ and thus prove the $p$-adic Artin conjecture in this case. One can extend this quite easily to the case when $\psi \rho$ is of type $S$, where $\rho$ is a one-dimensional Artin character such that $k_{\rho} \subseteq k_{\infty}$. (Such Artin characters $\rho$ will be said to be of type $W$.) However it is not difficult to find Artin characters $\psi$ not satisfying the above conditions. We can still deduce the $p$-adic Artin conjecture for such $\psi$ from the main conjecture, but the argument is somewhat more ad hoc.

Received May 6, 1981.

* Supported in part by a National Science Foundation grant. 


\section{§1. Algebraically defined polynomials}

Let $\psi$ be a $p$-adic Artin character over $k$, that is, a character of a Galois representation on a vector space over $\Omega_{p}$. Here $\Omega_{p}$ is an algebraic closure of the field $\boldsymbol{Q}_{p}$ of $p$-adic numbers. Let $G=\operatorname{Gal}\left(k_{\psi} k_{\infty} / k\right)$. The character $\psi$ is of type $S$ if and only if $G \cong \Delta \times \Gamma$, where $\Delta=\operatorname{Gal}\left(k_{\psi} / k\right)$ and $\Gamma=\mathrm{Gal}\left(k_{\infty} / k\right)$. The isomorphism arises from the restriction maps from $G$ onto $\Delta$ and $\Gamma$. Let $L$ denote the maximal abelian pro-p-extension of $k_{\psi} k_{\infty}$ in which only primes over $p$ are ramified. Let $X=\mathrm{Gal}\left(L / k_{\psi} k_{\infty}\right)$. The group $G$ acts on $X$ by $x \rightarrow \bar{g} x \bar{g}^{-1}$ for $x \in X$ and $g \in G$, where $\bar{g}$ denotes any extension of $g$ to an automorphism of $L$. Now since $k_{\psi}$ is totally real, $X$ is a torsion and noetherian $\Lambda$-module, where $\Lambda$ is the completed group ring of $\Gamma$ over $Z_{p}$. (See [9].) This implies that $V=X \otimes_{z_{p}} \Omega_{p}$ is a finite dimensional vector space over $\Omega_{p}$. Also $G$ acts on $V$ (through the first factor). Now assume that $\psi$ is irreducible. Let $e_{\psi}$ denote the idempotent element in the group ring $\Omega_{p}[\Delta]$ corresponding to $\psi$. Let $V_{\psi}$ $=e_{\psi} V$. As a representation space for $\Delta, V_{\psi}$ is a direct sum of irreducible representations with character $\psi$. Now $V_{\psi}$ is invariant under the action of $\Gamma$. If we let $\gamma_{0}$ be a fixed topological generator for $\Gamma$, then each root in $\Omega_{p}$ of the characteristic polynomial $c_{\psi}(T)$ of $\gamma_{0}-\mathrm{id}$. acting on $V_{\psi}$ occurs with multiplicity divisible by $d_{\psi}=\psi(1)$, the degree of the irreducible character $\psi$. Define $h_{\psi}(T)$ to be the monic polynomial such that $h_{\psi}(T)^{d_{\psi}}$ $=c_{\psi}(T)$.

We first want to show that $h_{\psi}(T)$ is unchanged by "liftings" of $\psi$. Suppose $k^{\prime}$ is a finite Galois extension of $k$ such that $k_{\psi} \subseteq k^{\prime}$ and $k^{\prime} \cap k_{\infty}$ $=k$. Let $\Delta^{\prime}=\mathrm{Gal}\left(k^{\prime} \mid k\right)$ and let $\psi^{\prime}$ be the character of $\Delta^{\prime}$ obtained from $\psi$ by composition with the restriction map $\Delta^{\prime} \rightarrow \Delta$. Let $V_{\psi^{\prime}}$ and $h_{\psi^{\prime}}(T)$ be defined just as above. Then we have the following proposition.

Proposition 1. $h_{\psi^{\prime}}(T)=h_{\psi}(T)$.

Proof. We will show that in fact $V_{\psi^{\prime}} \cong V_{\psi}$ as representation spaces for $\Gamma$. Let $L^{\prime}$ be the maximal abelian $p$-ramified pro-p-extension of $k^{\prime} k_{\infty}$ and let $X^{\prime}=\operatorname{Gal}\left(L^{\prime} \mid k^{\prime} k_{\infty}\right)$. We have $F \subseteq F^{\prime} \subseteq L^{\prime}$ and $L \subseteq L^{\prime}$, where $F=$ $k_{\psi} k_{\infty}, F^{\prime}=k^{\prime} k_{\infty}$. Let $H=\mathrm{Gal}\left(F^{\prime} \mid F\right)$. The image of $X^{\prime}$ under the natural homomorphism $X^{\prime} \rightarrow X$ is of finite index in $X$. This natural homomorphism factors through $X^{\prime} / I_{H} X^{\prime}$, where $I_{H}$ denotes the augmentation ideal in $Z_{p}[H]$. Let $a^{\prime}$ and $a$ denote the dimensions over $\boldsymbol{Q}_{p}$ of $\left(X^{\prime} / I_{H} X^{\prime}\right) \otimes_{z_{p}} \boldsymbol{Q}_{p}$ and $X \otimes_{z_{p}} Q_{p}$. Then $a^{\prime} \geq a$. Also there is a subfield $L_{0}^{\prime}$ of $L^{\prime}$ such that 
$F^{\prime} \subseteq L_{0}^{\prime} \subseteq L^{\prime}, X_{0}^{\prime}=\mathrm{Gal}\left(L_{0}^{\prime} / F^{\prime}\right) \cong Z_{p}^{a^{\prime}}$, and $L_{0}^{\prime}$ is contained in the fixed field for $I_{H} X^{\prime}$. Now Gal $\left(L_{0}^{\prime} / F\right)$ is a central extension of $X_{0}^{\prime}$ by $H$ and the transfer homomorphism maps $\operatorname{Gal}\left(L_{0}^{\prime} / F\right)$ onto a subgroup of $X_{0}^{\prime}$ containing $\left(X_{0}^{\prime}\right)^{h}$, where $h=|H|$. Thus $\mathrm{Gal}\left(L_{0}^{\prime} \mid F\right)$ has a quotient group isomorphic to $Z_{p}^{a^{\prime}}$. The field extension $L_{00}^{\prime} / F$ corresponding to this quotient group is unramified outside $p$ (since the inertia groups for such primes have finite order dividing $h$ ). Thus $L_{00}^{\prime} \subseteq L$ and hence $a^{\prime} \leq a$. Letting $V^{\prime}=X^{\prime} \otimes_{z_{p}} \Omega_{p}$, we have an isomorphism $V^{\prime} / I_{H} V^{\prime} \cong V$ induced from the map $X^{\prime} \rightarrow X$. Since $e_{\psi^{\prime}} V^{\prime}$ is mapped to $e_{\psi} V$ and since $e_{\psi^{\prime}} I_{H} V^{\prime}=\{0\}$, we have $e_{\psi^{\prime}} V^{\prime} \cong e_{\psi} V$ (as $\Gamma$-spaces), proving Proposition 1.

There is now no need to assume that our characters are faithful. We also extend the definition of $h_{\psi}(T)$ to reducible characters by $h_{\theta+\eta}(T)=$ $h_{\theta}(T) h_{\eta}(T)$. Let $K$ be a finite totally real Galois extension of $k$ such that $K \cap k_{\infty}=k$. Let $\Delta=\operatorname{Gal}(K / k)$ and let $\Delta_{0}$ be a subgroup of $\Delta$. Let $\psi$ be a character of $\Delta_{0}$ and let $\psi^{*}=\operatorname{Ind}_{\Delta_{0}}^{\Delta}(\psi)$.

Proposition 2. $h_{\psi *}(T)=h_{\psi}(T)$.

Proof. Let $L$ be the maximal $p$-ramified abelian pro-p-extension of $K k_{\infty}$ so that as before $V=\operatorname{Gal}\left(L / K k_{\infty}\right) \otimes_{z_{p}} \Omega_{p}$ is a representation space for $\operatorname{Gal}\left(K k_{\infty} / k\right) \cong \Delta \times \Gamma$ (canonically). If $\chi$ is either a character of $\Delta$ or of $\Delta_{0}$, we can of course define both $V_{\chi}$ and $h_{x}(T)$. Let $\alpha \in \Omega_{p}$. Consider $V_{\alpha}=\left\{v \in V \mid\left(\left(\gamma_{0}-\mathrm{id} \text {. }\right)-\alpha\right)^{t} v=0\right.$ for some $\left.t\right\}$. $V_{\alpha}$ is invariant under the action of $\Delta$. Let $\varphi_{\alpha}$ denote the character of the representation of $\Delta$ on $V_{\alpha}$. If $\chi$ is an irreducible character of either $\Delta$ or $\Delta_{0}$, then $\chi$ occurs in $V_{\alpha}$ with multiplicity $\left(\chi, \varphi_{\alpha}\right)_{\Delta}$ or $\left(\chi,\left.\varphi_{\alpha}\right|_{\Delta_{0}}\right)_{\Delta_{0}}$. Clearly this multiplicity will also be the multiplicity of $\alpha$ as a root of $h_{\chi}(T)$. If $\chi$ is reducible, the multiplicity of $\alpha$ as a root of $h_{x}(T)$ is also given by the above inner products. The proposition then follows immediately from the Frobenius Reciprocity Law $\left(\psi^{*}, \varphi_{\alpha}\right)_{\Delta}=\left(\psi,\left.\varphi_{\alpha}\right|_{\Delta_{0}}\right)_{\Delta_{0}}$.

Now we consider two Artin characters $\psi_{1}$ and $\psi_{2}$ over $k$, both of type $S$, such that $\psi_{1}=\psi_{2} \rho$, where $\rho$ is of type $W$. If $f(T)$ and $g(T)$ are two polynomials, we write $f(T) \sim g(T)$ when $f(T)=u g(T)$ with $u \in \Omega_{p}$ of absolute value 1 . Then we have

Proposition 3. Let $\rho\left(\gamma_{0}\right)=\rho_{0}$. Then $h_{\psi_{1}}(T) \sim h_{\psi_{2}}\left(\rho_{0}(1+T)-1\right)$.

Proof. We can assume that $\psi_{1}$ and $\psi_{2}$ are irreducible. Let $K_{i}=k_{\psi_{i}}$ and $\Delta_{i}=\operatorname{Gal}\left(K_{i} / k\right)$ for $i=1,2$. Obviously, $K_{1} k_{\infty}=K_{2} k_{\infty}=F$, say. After 
identifying $\Delta_{1}$ and $\Delta_{2}$ with the corresponding subgroups of $\mathrm{Gal}(F / k)$, we actually have $\Delta_{1}=\Delta_{2}=\operatorname{Gal}\left(F / k_{\infty}\right)$. Regarding $\psi_{1}$ and $\psi_{2}$ as characters of $\mathrm{Gal}(F / k)$, their restrictions to $\mathrm{Gal}\left(F / k_{\infty}\right)$ coincide. Hence $V_{\psi_{1}}=V_{\psi_{2}}$. Let $\gamma_{1}$ and $\gamma_{2}$ be the topological generators of $\Gamma_{1}=\operatorname{Gal}\left(F / K_{1}\right)$ and $\Gamma_{2}=$ $\operatorname{Gal}\left(F / K_{2}\right)$ such that $\left.\gamma_{1}\right|_{k_{\infty}}=\gamma_{0}$. Clearly $\gamma_{2}=\gamma_{1} \delta$ for some $\delta \in \operatorname{Gal}\left(F / k_{\infty}\right)$. Actually $\delta$ must be in the center of $\mathrm{Gal}\left(F / k_{\infty}\right)$. It is easy to determine $\delta$ since we must have $\gamma_{2} \in \operatorname{ker}\left(\psi_{2}\right)$. Thus

$$
d_{\psi_{2}}=\psi_{2}\left(\gamma_{2}\right)=\psi_{1} \rho^{-1}\left(\gamma_{1} \delta\right)=\psi_{1}\left(\gamma_{1} \delta\right) \rho^{-1}\left(\gamma_{1} \delta\right)=\psi_{1}(\delta) \rho^{-1}\left(\gamma_{1}\right)=\psi_{1}(\delta) \rho_{0}^{-1}
$$

and therefore $\psi_{1}(\delta)=d_{\psi_{2}} \rho_{0}=d_{\psi_{1}} \rho_{0}$, which determines $\delta$ since $\psi_{1}$ is faithful on $\operatorname{Gal}\left(F / k_{\infty}\right)$. Clearly $\delta$ acts on $V_{\psi_{1}}=V_{\psi_{2}}$ as multiplication by $\rho_{0}$. Proposition 3 follows immediately.

Now let $\psi$ be an irreducible character of type $S$. If $\alpha \in \Omega_{p}$, let $A$ denote $\left(\gamma_{0}-\right.$ id.) $-\alpha$ acting on $V_{\psi}$. Clearly, $\operatorname{ker}\left(A^{t}\right) / \operatorname{ker}\left(A^{t-1}\right)$ has dimension over $\Omega_{p}$ divisible by $d_{\psi}$ for all $t \geq 1$. Also, under certain assumptions on $k_{\psi}$, it is possible to show that $V$ has a cyclic vector for the action of $\mathrm{Gal}\left(k_{\psi} k_{\infty} / k\right)$. For example, this would be true if $k=\boldsymbol{Q}$, the $p$-adic completions of $k_{\psi}$ contained no primitive $p$-th (or 4 th if $p=2$ ) roots of unity, and Iwasawa's $\lambda$-invariant $\lambda\left(k_{\psi}\right)$ vanished. If $V_{\psi}$ is cyclic for $\operatorname{Gal}\left(k_{\psi} k_{\infty} / k\right)$, then $\operatorname{ker}\left(A^{t}\right) / \operatorname{ker}\left(A^{t-1}\right)$ would be cyclic for the action of $\Delta$ and would therefore have dimension over $\Omega_{p}$ at most $d_{\psi}^{2}$. Let $m_{\psi}(T)$ denote the minimal polynomial for $\gamma_{0}-$ id. acting on $V_{\psi}$. The above observations lead to the following result, which is closely related to Theorem 3 of [10].

Proposition 4. $\quad V_{\psi}$ is annihilated by $h_{\psi}\left(\gamma_{0}-\mathrm{id}\right.$.). Hence $m_{\psi}(T)$ divides $h_{\psi}(T)$. If $V_{\psi}$ has a cyclic vector for the action of $\mathrm{Gal}\left(k_{\psi} k_{\infty} / k\right)$, then $h_{\psi}(T)$ divides $m_{\psi}(T)^{d \psi}$.

\section{§2. $p$-adic Artin $L$-functions}

First let $\psi$ be a 1-dimensional $\Omega_{p}$-valued Artin character over $k$. We still assume that $k_{\psi}$ is totally real. The existence of the $p$-adic $L$-function $L_{p}(s, \psi)$ was first established by Deligne and Ribet. (See [4] and also [11].) Alternative approaches were given later by Barsky [1] and Cassou-Nogues [2]. Let $\sigma: \Omega_{p} \rightarrow C$ be a fixed isomorphism. Then $\sigma \circ \psi$ is a complex-valued 1-dimensional Artin character. Let $L^{*}(z, \sigma \circ \psi)$ be the corresponding Artin $L$-function with all Euler factors for primes dividing $p$ omitted. The values $L^{*}(1-n, \sigma \circ \psi)$ are in $\boldsymbol{Q}$ (values of $\left.\sigma \circ \psi\right)$ and are nonzero for even $n$. Let $L^{*}(1-n, \psi)=\sigma^{-1}\left(L^{*}(1-n, \sigma \circ \psi)\right)$, which is in fact independent of the 
choice of $\sigma$. The function $L_{p}(s, \psi)$ can be characterized as the unique, continuous, $\Omega_{p}$-valued function on $Z_{p}$ such that $L_{p}(1-n, \psi)=L^{*}(1-n, \psi)$ for all positive $n \equiv 0(\bmod p-1)(\operatorname{or}(\bmod 2)$ if $p=2)$. It is defined and analytic (except for at most a simple pole at $s=1$ when $\psi$ equals the principal character $\psi^{0}$ ) in a disc in $\Omega_{p}$ about zero of radius greater than 1. $L_{p}(s, \psi)$ is not identically zero.

Now assume $\psi$ is an $\Omega_{p}$-valued Artin character of arbitrary degree (with $k_{\psi}$ still totally real). Let $\Delta=\operatorname{Gal}(K / k)$ where $K$ is a finite, totally real Galois extension of $k$ containing $k_{\psi}$. By Brauer's Induction Theorem,

$$
\psi=\sum_{i=1}^{t} a_{i} \psi_{i}^{*}
$$

where $a_{i} \in \boldsymbol{Z}$ and $\psi_{i}$ is a 1-dimensional Artin character of a subgroup $\Delta_{i}$ of $\Delta$ for $i=1, \cdots, t$. We define the $p$-adic Artin $L$-function for $\psi$ by

$$
L_{p}(s, \psi)=\sum_{i=1}^{t} L_{p}\left(s, \psi_{i}\right)^{a_{i}} .
$$

It is clear that $L_{p}(1-n, \psi)=\sigma^{-1}\left(L^{*}(1-n, \sigma \circ \psi)\right)$ for $n>0, n \equiv 0(\bmod$ $p-1$ or 2$)$, where $L^{*}(z, \sigma \circ \psi)$ is the complex Artin $L$-function with Euler factors for primes over $p$ removed. $L_{p}(s, \psi)$ is therefore uniquely determined and is independent of (1). It of course has the basic properties: $L_{p}(s, \theta+\eta)=L_{p}(s, \theta) L_{p}(s, \eta), L_{p}\left(s, \psi^{\prime}\right)=L_{p}(s, \psi)$, and $L_{p}\left(s, \psi^{*}\right)=L_{p}(s, \psi)$. Here the notation is as in the previous section. $L_{p}(s, \psi)$ will be meromorphic on some disc about zero in $\Omega_{p}$.

Let $k^{\prime}=k\left(\mu_{p}\right)$ (or $k\left(\mu_{4}\right)$ if $p=2$ ), where $\mu_{n}$ denotes the $n$-th roots of unity in a fixed algebraic closure of $k$. Now $k_{\infty}^{\prime}$ contains all $p$-power roots of unity. The action of $\Gamma=\operatorname{Gal}\left(k_{\infty} / k\right) \cong \operatorname{Gal}\left(k_{\infty}^{\prime} / k^{\prime}\right)$ on $p$-power roots of unity is given by a homomorphism $\kappa: \Gamma \rightarrow Z_{p}^{\times}$. Let $\kappa_{0}=\kappa\left(\gamma_{0}\right)$. Note that $\kappa_{0} \equiv 1\left(\bmod p^{r}\right)$ if $\mu_{p^{r}} \subseteq k^{\prime}$.

If $\psi$ is a 1-dimensional Artin character for $k$, then there exists a power series $G_{\psi}(T)$ with coefficients in $\mathcal{O}_{\psi}=Z_{p}$ [values of $\psi$ ] such that

$$
L_{p}(1-s, \psi)=\frac{G_{\psi}\left(\kappa_{0}^{s}-1\right)}{H_{\psi}\left(\kappa_{0}^{s}-1\right)}
$$

for all $s \in Z_{p}$ (except $s=0$ if $\left.\psi=\psi^{0}\right)$. Here $H_{\psi}(T)=\xi(1+T)-1$ if $\psi$ is of type $W$, where $\xi=\psi\left(\gamma_{0}\right)$, and $H_{\psi}(T)=1$ if $\psi$ is not of type $W$. Also, if $\rho$ is of type $W$, then we have

$$
G_{\psi \rho}(T)=G_{\psi}\left(\rho_{0}(1+T)-1\right) \quad \text { and } \quad H_{\psi \rho}(T)=H_{\psi}\left(\rho_{0}(1+T)-1\right)
$$


where $\rho_{0}=\rho\left(\gamma_{0}\right)$. (See [11] for an explanation.) As in chapter 6 of [8], one can use (3) to show that $L_{p}(s, \psi)$ is an analytic function in a certain disc about zero.

We can derive a similar expression for $L_{p}(s, \psi)$ where $\psi$ is of arbitrary degree by using (1), (2), and (3). However note that if $f$ is an extension of $k$ (corresponding to a subgroup of 4$)$, then $\mathrm{Gal}\left(f_{\infty} / f\right)$ will be identified with a subgroup of $\Gamma$ of finite index. A topological generator will be a power of $\gamma_{0}$. The $p$-adic $L$-functions for 1-dimensional Artin characters over $f$ will have expressions similar to (3), but with possibly different arguments. We will however always write the power series and polynomials involved in terms of $T=\kappa_{0}^{s}-1$. (This is clearly possible.) Define $H_{\psi}(T)=\prod_{i=1}^{t} H_{\psi_{i}}(T)^{a_{i}}$, where the $\psi_{i}$ 's and $a_{i}$ 's are as in (1). It is not a difficult exercise to show that $H_{\psi}(T)$ is well-defined. In fact, if $\psi$ is 1 dimensional, $H_{\psi}(T)$ will be just as defined above. If $\psi$ is irreducible of degree greater than 1 , then $H_{\psi}(T)=1$. We also define $G_{\psi}(T)=\prod_{i=1}^{t} G_{\psi_{i}}(T)^{a_{i}}$, a quotient of power series with coefficients in the integers of a finite extension of $\boldsymbol{Q}_{p}$. Then (3) will hold for all $s$ in some disc containing $Z_{p}$ except possibly for finitely many values of $s$. Consequently, $G_{\psi}(T)$ depends only on $\psi$ (and on the choice of topological generator $\gamma_{0}$ of $\Gamma$ ). We can now formulate one of the two conjectures mentioned in the introduction.

p-adic Artin conjecture: $G_{\psi}(T) \in Z_{p}[[T]] \otimes_{z_{p}} \Omega_{p}$.

Some remarks are in order. It is easy to see that $G_{\psi}(T)$ is in the quotient field of $\mathcal{O}_{\psi}[[T]]$. Thus the above conjecture amounts to the statement that $c G_{\psi}(T) \in \mathcal{O}_{\psi}[[T]]$ for some nonzero $c \in \mathcal{O}_{\psi}$. A stronger form of the above conjecture would be that $G_{\psi}(T)$ itself is in $\mathcal{O}_{\psi}[[T]]$. We will discuss this question later. Also the $p$-adic Artin conjecture implies that if $\psi$ does not have $\psi^{0}$ as a constituent, then $L_{p}(s, \psi)$ is in fact defined and holomorphic in the disc $|s|<r$ where $r=\left|\kappa_{0}-1\right|^{-1}|p|^{1 /(p-1)}$. (See the end of chapter 6 of [8] for an explanation of this point.) However this last statement is not equivalent to the conjecture.

By the Weierstrass Preparation Theorem, we can uniquely write $G_{\psi}(T)$ in the form $G_{\psi}(T)=\pi^{m} u(T) g_{\psi}(T)$, where $\pi$ is a uniformizing parameter, $m \in Z, u(T)$ is an invertible element of $\mathcal{O}_{\psi}[[T]]$, and $g_{\psi}(T)=g^{\prime}(T) / g^{\prime \prime}(T)$, where $g^{\prime}$ and $g^{\prime \prime}$ are monic polynomials in $\mathcal{O}_{\psi}[T]$ whose nonleading coefficients are divisible by $\pi$. We define $m_{\psi}=m / e$ and $\ell_{\psi}=\operatorname{deg}\left(g^{\prime}\right)-\operatorname{deg}\left(g^{\prime \prime}\right)$. Here $e$ denotes the ramification index for the extension $\boldsymbol{Q}_{p}(\psi) / \boldsymbol{Q}_{p}$. 
We close this section by simply listing several easily proved properties.

(i) Let $\theta$ and $\eta$ be Artin characters over $k$. $G_{\theta+\eta}(T)=G_{\theta}(T) G_{\eta}(T), H_{\theta+\eta}(T)=H_{\theta}(T) H_{\eta}(T), g_{\theta+\eta}(T)=g_{\theta}(T) g_{\eta}(T)$, $m_{\theta+\eta}=m_{\theta}+m_{\eta}, \ell_{\theta+\eta}=\ell_{\theta}+\ell_{\eta}$

(ii) Let $\psi_{1}=\psi_{2} \rho$ where $\rho$ is of type $W$. Let $\rho_{0}=\rho\left(\gamma_{0}\right)$.

Then $G_{\psi_{1}}(T)=G_{\psi_{2}}\left(\rho_{0}(1+T)-1\right), H_{\psi_{1}}(T)=H_{\psi_{2}}\left(\rho_{0}(1+T)-1\right)$, $g_{\psi_{1}}(T) \sim g_{\psi_{2}}\left(\rho_{0}(1+T)-1\right), m_{\psi_{1}}=m_{\psi_{2}}, \ell_{\psi_{1}}=\ell_{\psi_{2}}$.

(As before, $\sim$ means the two rational functions differ by a factor in $\Omega_{p}$ of absolute value 1 .)

\section{§3. The Main Conjecture}

We now state the main conjecture for the $p$-adic $L$-functions constructed by Deligne and Ribet.

MaIn Conjecture. Let $\psi$ be a 1-dimensional Artin character of type S. Then $h_{\psi}(T)=g_{\psi}(T)$.

Note that this is slightly different than the usual version of the main conjecture, which is in terms of the (-1)-eigenspace for complex conjugation in a vector space constructed from an inverse limit of ideal class groups. The above statement is in fact equivalent. The equivalence is discussed in detail in [6] for $k=\boldsymbol{Q}$, but the arguments there extend without change to the more general case. Note also that if $\rho$ is a character of type $W$ and if $\psi \rho$ is also of type $S$, then the main conjecture for $\psi$ is equivalent to the main conjecture for $\psi \rho$ by Proposition 3.

Now let $K$ be a finite, totally real Galois extension of $k$ such that $K \cap k_{\infty}=k$, and let $\Delta=\mathrm{Gal}(K / k)$. Let $\theta$ be a 1-dimensional character of a subgroup $\Delta_{0}$ of $\Delta$. If $k_{0}$ is the fixed field for $\Delta_{0}$, then clearly, the choice of $\gamma_{0}$ and $\kappa_{0}$ for the new base field $k_{0}$ can be the same as for $k$. We will then have $g_{\theta}(T)=g_{\theta *}(T)$, where, as before, $\theta^{*}=\operatorname{Ind}_{\Delta_{0}}^{\Delta}(\theta)$. By proposition 2, we also have $h_{\theta}(T)=h_{\theta *}(T)$. If we assume that the main conjecture is true for all such $\theta$, then, by Brauer's Induction Theorem, we get the following consequence: If $\psi$ is a character of $\Delta$ of arbitrary degree, then $h_{\psi}(T)=g_{\psi}(T)$ and hence $g_{\psi}(T)$ is in fact a polynomial. Therefore, the main conjecture implies the $p$-adic Artin conjecture for Artin characters $\psi$ of type $S$. Indeed, a "main conjecture for characters of type $S$ " would be true. The general case is dealt with in the next proposition. 
Proposition 5. The main conjecture implies the p-adic Artin conjecture.

Proof. Let $\psi$ be an Artin character of arbitrary degree for $k$. The base fields for which we will assume the main conjecture will be subfields of $k_{\psi}$.

First assume that $\psi \rho$ is of type $S$ for some $\rho$ of type $W$. Until now, the polynomial $h_{\psi}(T)$ has been defined only when $\psi$ is of type $S$. If $\psi \rho$ is of type $S$, we define $h_{\psi}(T)$ to be the monic polynomial satisfying $h_{\psi}(T)$ $\sim h_{\psi \rho}\left(\rho_{0}^{-1}(1+T)-1\right)$, where $\rho_{0}=\rho\left(\gamma_{0}\right)$. By Proposition $3, h_{\psi}(T)$ is well defined. Now, by the discussion preceding Proposition 5 and by (ii) of the previous section, it is clear that $g_{\psi}(T)=h_{\psi}(T)$ and hence $g_{\psi}(T)$ is a polynomial in this case too.

Let $\psi$ be arbitrary. Let $K=k_{\psi}$ and let $\Delta=\operatorname{Gal}(K / k)$. If $f$ is an extension of $k$ in $K$ and if $\theta$ is a 1-dimensional Artin character over $f$, then obviously $\theta \rho$ is of type $S$ for some $\rho$ of type $W$ over $f$. Thus $h_{\theta}\left(T_{e}\right)$ is defined. Here $T_{e}=(1+T)^{p e}-1$, where $p^{e}=\left[f \cap k_{\infty}: k\right]$. (The role of $\kappa_{0}$ and $\gamma_{0}$ is played by $\kappa_{0}^{p e}$ and $\gamma_{0}^{p e}$ over $f$.) We define $h_{\theta^{*}}(T)=h_{\theta}\left(T_{e}\right)$, where $\theta^{*}=\operatorname{Ind}_{\text {Gal }(k / f)}^{4}(\theta)$. If $\psi=\sum_{i=1}^{t} a_{i} \psi_{i}^{*}$ as in (1), we then define a rational function $h_{\psi}(T)$ by $h_{\psi}(T)=\prod_{i=1}^{t} h_{\psi_{i}^{*}}(T)^{a_{i}}$. We will show (unconditionally) that $h_{\psi}(T)$ is well-defined and is in fact a polynomial. The proposition follows from this because the main conjecture implies that $g_{\psi}(T)=h_{\psi}(T)$.

Now let $\alpha \in \Omega_{p}$. We will prove that the multiplicity of $\alpha$ in $h_{\psi}(T)$ depends only on $\psi$ and $\alpha$ and is nonnegative. Put $F=K k_{\infty}$ and $L=$ the maximal abelian pro-p-ramified extension of $F$. If $G=\mathrm{Gal}(F / k)$, then Gal $(L / F) \otimes_{z_{p}} \Omega_{p}$ is, as before, a finite dimensional space for $G$ over $\Omega_{p}$. We let $V$ be the semisimplification of the above representation of $G$. Letting $N=\mathrm{Gal}\left(F / k_{\infty}\right)$, we have an exact sequence $1 \rightarrow N \rightarrow G \rightarrow \Gamma \rightarrow 1$ which is in fact split. We can choose $g_{0} \in G$ such that $\left.g_{0}\right|_{k \infty}=\gamma_{0}$ that such and $g_{0}$ is a topological generator of a complement for $N$ in $G$. We let

$$
V_{\alpha}=\left\{v \in V \mid\left(g_{0}^{p^{r}}-(1+\alpha)^{p r}\right) v=0 \text { for some } r\right\} .
$$

$V_{\alpha}$ is a $G$-invariant subspace which does not depend on our choice of $g_{0}$. Now we have a homomorphism $\chi_{\alpha}: G \rightarrow \Omega_{p}^{\times}$which factors through $\Gamma$ and sends $g_{0}$ to $1+\alpha$. We let $W_{\alpha}=V_{\alpha} \otimes \chi_{\alpha}^{-1}$ so that $G$ acts on $W$ through a finite quotient group $\bar{G}=G / H$. We may assume that $K$ is contained in the fixed field for $H$. Then $\Delta$ is a homomorphic image of $\bar{G}$ and all the characters we are considering can be viewed as characters of $\bar{G}$ or 
subgroups of $\bar{G}$. Let $\varphi_{\alpha}$ be the character of the representation of $\bar{G}$ on $W_{\alpha}$.

Consider an arbitrary field $f$ such that $k \subseteq f \subseteq K$. Let $\Delta_{f}=\mathrm{Gal}(K / f)$ and let $\bar{G}_{f}$ be the inverse image of $\Delta_{f}$ in $\bar{G}$. Let $\theta$ be a 1 -dimensional character of $\Delta_{f}$. Assume $\left[f \cap k_{\infty}: k\right]=p^{e}$ and let $T_{e}=(1+T)^{p e}-1$ as before. For some character $\rho$ of type $W$ for $f, \eta=\theta \rho$ will be of type $S$ and so $\operatorname{Gal}\left(f_{\theta} f_{\infty} / f\right)=\operatorname{Gal}\left(f_{\eta} f_{\infty} / f\right)$ will be canonically isomorphic to $\operatorname{Gal}\left(f_{\eta} \mid f\right) \times \Gamma_{f}$, where $\Gamma_{f}=\operatorname{Gal}\left(f_{\infty} / f\right)$. Let $\gamma_{f} \in \Gamma_{f}$ be such that $\left.\gamma_{f}\right|_{k_{\infty}}=\gamma_{0}^{p e}$. It is easy to see that the multiplicity of $\alpha_{e}=(1+\alpha)^{p e}-1$ as a root of $h_{\eta}\left(T_{e}\right)$ is given by $\left(\left.\varphi_{\alpha}\right|_{\bar{G}_{f}}, \eta\right)_{\bar{G}_{f}}$. (We can assume that $H$ is chosen so that $\eta$ factors through $\bar{G}_{f}$. Also, see the argument used to prove Proposition 1.) Let $\xi=\rho\left(\gamma_{f}\right)^{-1}$. Choose $\beta \in \Omega_{p}$ so that $(1+\beta)^{p e}=\xi(1+\alpha)^{p e}$. Clearly $V_{\beta}=V_{\alpha}$ and $\left.\varphi_{\beta}\right|_{\bar{G}_{f}}=\left(\left.\varphi_{\alpha}\right|_{\bar{G}_{f}}\right) \cdot \rho$. The multiplicity of $\beta_{e}=(1+\beta)^{p e}-1$ as a root of $h_{\eta}\left(T_{e}\right)$ equals $\left(\left.\varphi_{\beta}\right|_{\bar{G}_{f}}, \eta\right)_{\bar{G}_{f}}=\left(\left.\varphi_{\alpha}\right|_{\bar{G}_{f}}, \eta \rho^{-1}\right)_{\bar{G}_{f}}=\left(\left.\varphi_{\alpha}\right|_{\bar{G}_{f}}, \theta\right)_{\bar{G}_{f}}$. The multiplicity of $\beta_{e}$ as a root of $h_{\eta}\left(T_{e}\right)$ is also the multiplicity of $\alpha_{e}$ as a root of $h_{\theta}\left(T_{e}\right)$. This in turn equals the multiplicity of $\alpha$ as a root of $h_{\theta *}(T)$. To summarize, $\alpha$ has multiplicity $\left(\left.\varphi_{\alpha}\right|_{\bar{G}_{f}}, \theta\right)_{\bar{G}_{f}}=\left(\varphi_{\alpha}, \theta^{*}\right)_{\bar{G}}$ in the polynomial $h_{\theta *}(T)$. Applying this to $\theta=\psi_{i}$ for $i=1, \cdots, t$, we see that $\alpha$ has (non-negative) multiplicity $\left(\varphi_{\alpha}, \psi\right)_{\bar{G}}$ in $h_{\psi}(T)$, proving that $h_{\psi}(T)$ is indeed a polynomial.

Q.E.D.

\section{§4. Some questions}

The $p$-adic Artin conjecture would seem to be more approachable than the complex Artin conjecture in some ways. For a given $\psi$ and $p$, it might quite conceivably be possible to verify the $p$-adic Artin conjecture by a purely computational approach. For example, one might be able to eliminate possible poles by using different representations of $L_{p}(s, \psi)$ in the form of (2). On the other hand, there is a rich circle of connections between the complex Artin conjecture and the theory of modular forms and representation theory. These connections have led to proofs of the complex Artin conjecture for some non-monomial characters. It would be interesting to find similar connections in the $p$-adic case. Can the $p$-adic Artin conjecture be related directly to the complex conjecture? Of course, both are valid for monomial characters.

Behavior at $s=1$. For 1-dimensional Artin characters $\psi$, it should be true that $L_{p}(s, \psi)$ has a pole (which must be simple) at $s=1$ when $\psi=\psi^{0}$ and is nonzero at $s=1$ when $\psi \neq \psi^{0}$. The same result would 
then follow for irreducible Artin characters $\psi$ of arbitrary degree. It would be interesting to know more about the values at $s=1$. There is a $p$-adic Stark conjecture (formulated by Serre). Now our proof of Proposition 5 is based on the conjectured properties of the representation space $V$. If we could somehow construct a similar representation space (perhaps by proving a weak form of a $p$-adic Stark conjecture for Deligne-Ribet $p$-adic $L$-functions), it might be possible to prove the $p$-adic Artin conjecture unconditionally. It would be interesting to examine exactly what is involved in constructing such a representation space.

Behavior at $s=0$. This topic has been discussed in some detail by Gross in [7]. Let $\omega$ be the Teichmuller character over $k$ (i.e. the 1-dimensional $\Omega_{p}$-valued Artin character over $k$ giving the Galois action on $p$-th or 4th roots of unity). If $\psi$ is an arbitrary Artin character, we define $\psi_{n}=\psi \omega^{-n}$ for every integer $n$. For 1-dimensional even $\Omega_{p}$-valued characters $\psi$, we have for $n \geq 1$

$$
L_{p}(1-n, \psi)=\sigma^{-1}\left(L^{*}\left(1-n, \sigma \circ \psi_{n}\right)\right)
$$

and also these values are non-zero for $n \geq 2$. Using (2), one can easily extend (5) to the case when $\psi$ is of arbitrary degree provided $n \geq 2$. It would be interesting to prove that $L_{p}(s, \psi)$ is in fact analytic at $s=0$ and has the correct value. The order of vanishing at zero of $L_{p}(s, \psi)$ and $L^{*}\left(z, \sigma \circ \psi_{1}\right)$ should be the same too. If $\psi$ is 1-dimensional, let $a$ and $b$ denote the order of vanishing of $L_{p}(s, \psi)$ at $s=0$ and $L^{*}\left(z, \sigma \circ \psi_{1}\right)$ at $z=0$, respectively. Also, let $c$ be the order of vanishing of $h_{\psi}(T)$ at $T=\kappa_{0}-1$. The only relationships between $a, b$, and $c$ that seem to be known at present are the following: (i) If one is nonzero, they all are and (ii) $c \geq b$. (Proofs omitted.) Note that $b$ is easily described. It is simply the number of prime ideals $\mathfrak{p}$ of $k$ dividing $p$ such that $\psi_{1}(\mathfrak{p})=1$.

Let $\theta=\psi \rho$, where $\psi$ is an Artin character of arbitrary degree and $\rho$ is of type $W$. If $\rho$ is of sufficiently large order, then it is easy to see that in fact

$$
L_{p}(0, \theta)=\sigma^{-1}\left(L\left(0, \sigma \circ \theta_{1}\right)\right) .
$$

(The Euler factors for primes dividing $p$ will be trivial.) Let $\lambda_{\psi}$ denote the degree of $h_{\psi}(T)$. One can use (6), the arguments in section 5 of [5], and the arguments in section 2 of [6] to prove the following result: If $\psi$ is such that $\psi_{1}$ is rational valued, then $\ell_{\psi}=\lambda_{\psi}$. One could also relate 
$m_{\psi}$ to Iwasawa's $\mu$-invariant. If the well known conjecture that $\mu$ vanishes for the cyclotomic $Z_{p}$-extension of an arbitrary base field is true, then it would follow that $m_{\psi}=0$ if $p$ is odd and that $m_{\psi}=\psi(1)[k: Q]$ if $p=2$. One would first prove this for all $\psi$ such that $\psi_{1}$ is rational-valued, then for 1-dimensional $\psi$, and finally for $\psi$ of arbitrary degree. Thus, the vanishing of Iwasawa's $\mu$-invariant would imply that the $p$-adic Artin conjecture is equivalent to the stronger conjecture that $G_{\psi}(T) \in \mathcal{O}_{\psi}[[T]]$.

\section{REFERENCES}

[ 1 ] Barsky, D., Fonctions Zeta $p$-adiques d'une Classe de Rayon des Corps de Nombres Totalement-reels, Group d'etude d'analyse ultrametrique (1977-78).

[2] Cassou-Nogues, P., Valeurs aux entieres negatif des fonctions zeta et fonctions zeta $p$-adiques, preprint.

[ 3 ] Coates, J., p-adic L-functions and Iwasawa's theory, Alg. No. Theory, A. Frohlich, ed., Academic Press 1977.

[4] Deligne, P. and Ribet, K., Values of abelian $L$-functions at negative integers over totally real fields, Invent. Math., 59 (1980), 227-286.

[ 5 ] Greenberg, R., On $p$-adic $L$-functions and cyclotomic fields, Nagoya Math. J., 56 (1974), 61-77.

[ 6 ] - On $p$-adic $L$-functions and cyclotomic fields II, Nagoya Math. J., 67 (1977), 139-158.

[ 7 ] Gross, B., On the behavior of $p$-adic $L$-functions at $s=0$, preprint.

[8] Iwasawa, K., Lectures on $p$-adic L-functions, Ann. Math. Studies 74, Princeton University Press, 1972.

[9] - On $\boldsymbol{Z}_{l}$-extensions of algebraic numbers fields, Ann. of Math., 98 (1973), $246-326$.

[10] - On $p$-adic representations associated with $\boldsymbol{Z}_{p}$-extensions, preprint.

[11] Ribet, K., Report on $p$-adic $L$-functions over totally real fields, Asterisque, 61 (1979), 177-192.

Department of Mathematics

University of Washington

Seattle, Washington 98195

USA 